\title{
Can vitamin C and interleukin 6 levels predict preterm premature rupture of membranes: evaluating possibilities in North Indian population
}

\author{
Sumedha Gupta, MS ${ }^{1}$, Harsha S. Gaikwad, MD¹, Banashree Nath, MS², Achla Batra, DGO, DNB'1 \\ Department of Obstetrics and Gynaecology, ${ }^{1}$ VMMC and Safdarjung Hospital, New Delhi, ${ }^{2}$ All India Institute of Medical Sciences, Raebareli, India
}

\section{Objective}

We aimed to evaluate the relation between plasma ascorbic acid levels and the occurrence of preterm premature rupture of membranes (PPROM) and whether patients with ascorbic acid deficiency have predisposition to microbial invasion, as revealed by serum interleukin-6 (IL-6) levels and confirmed by placental culture sensitivity evaluation.

\section{Methods}

This controlled study involved 50 women with PPROM at gestational age between 28 and 36.6 weeks. Fifty patients with uncomplicated pregnancy and intact membranes were observed as control group. Serum ascorbic acid and IL-6 levels were evaluated for PPROM group subjects and controls, matched by their gestational age and body mass index. Repeat samples for IL- 6 were taken after 48 hours of admission in PROM group subjects and at the onset of labor in controls. Placental membranes were sent for culture sensitivity evaluation after delivery.

\section{Results}

The mean plasma level of ascorbic acid was $0.60 \pm 0.35 \mathrm{mg} / \mathrm{dL}$ and $1.18 \pm 0.43 \mathrm{mg} / \mathrm{dL}$ in the PPROM and control groups, respectively, showing significant difference. Plasma IL-6 level rose from $18.18 \pm 5.94 \mathrm{pg} / \mathrm{mL}$ to $34.32 \pm 7.31 \mathrm{pg} / \mathrm{mL}$ after 48 hours of admission in the PPROM group $(P=0.000)$. Plasma IL-6 level in controls rose from $5.59 \pm 4.36 \mathrm{pg} / \mathrm{mL}$ at admission to $7.36 \pm 3.67 \mathrm{pg} / \mathrm{mL}$ at the onset of labor. Membrane culture test results were positive in a total of 40 samples, of which 37 samples were from the PPROM group subjects.

\section{Conclusion}

Vitamin C deficiency, when complemented with elevated serum IL-6 level, can predict susceptibility to PPROM in the third trimester; the risk increases in women with prior history of similar pathology.

Keywords: Preterm premature rupture of fetal membranes; Ascorbic acid; Interleukin-6; Complications; Pregnancy

\section{Introduction}

Preterm premature rupture of membranes (PPROM) is the rupture of the chorion-amniotic membrane and leakage of amniotic fluid before onset of labor pains, prior to 37th week of pregnancy. Although PPROM involves $1-5 \%$ of pregnancies, it is responsible for $20-55 \%$ preterm deliveries [1]. PPROM is an important contributor to maternal and perinatal morbidity and mortality. The major cause of perinatal morbidity and mortality associated with PPROM is prematurity. Maternal sepsis is a rare but life-threatening complication reported in nearly $1 \%$ of cases [2].

It is postulated that mediators, released either from stretch-
Received: 2019.10.01. Revised: 2020.02.13. Accepted: 2020.02.25. Corresponding author: Banashree Nath, MS

Department of Obstetrics and Gynaecology, All India Institute of Medical Sciences, Dalmau Rd, Munshiganj, Raebareli 229405, India

E-mail: nathbanashree@gmail.com

https://orcid.org/0000-0001-5708-8769

Articles published in Obstet Gynecol Sci are open-access, distributed under the terms of the Creative Commons Attribution Non-Commercial License (http://creativecommons. org/licenses/by-nc/3.0/) which permits unrestricted non-commercial use, distribution, and reproduction in any medium, provided the original work is properly cited.

Copyright $\odot 2020$ Korean Society of Obstetrics and Gynecology 


\section{Obstetrics \& Gynecology Science}

Sumedha Gupta, et al. Predictability of vit C and IL 6 levels for PPROM

ing of membranes or infection, lead to activation of destructive enzymes in the matrix, thus causing rupture of amniotic membranes. Reactive oxygen species (ROS) is one of the factors involved in membrane destruction. Micronutrients stabilize the ROS, and hence, its deficiency leads to abnormal collagen structure synthesis that has been associated with increased risk of PPROM. The micronutrient Ascorbic acid (vitamin C) is an effective water-soluble antioxidant that scavenges several ROS, thus reducing oxidative stress [3]. It participates in the equilibrium between synthesis and degradation of collagen and this may be critical in reducing the occurrence of PPROM as well. Interleukin-6 (IL-6) is one of the inflammatory cytokines produced by macrophages, amnion, chorion, and decidual cells that is elevated in maternal serum and amniotic fluid in patients with PPROM with microbial invasion, even before the development of signs and symptoms of chorioamnionitis. A meta-analysis showed that IL-6 might be a sensitive and specific diagnostic marker for the early diagnosis of neonatal sepsis with premature rupture of membranes (PROM) [4]. Hence measuring IL-6 cytokine in maternal serum is a simpler and less invasive method of predicting impending infection in PPROM [5].

The present case-control study was conducted to investigate whether there is a relation of plasma ascorbic acid levels to the occurrence of PPROM and if those with deficiency have a significant predisposition to microbial invasion of amniotic membranes, as revealed by elevated IL- 6 levels. Our aim was also to determine fetal morbidity and mortality in patients with PPROM and co-relate the outcome with serum vitamin C and IL-6 levels.

\section{Materials and methods}

This was a case control study conducted in a tertiary level hospital in Northern India for a period of 18 months. The study group subjects were hospitalized patients with PPROM. Control group subjects were patients from pre-natal clinics, identified during routine checkup.

Osaikhuwuomwan et al. [6] reported that 33 subjects in a study group and 21 in a control group had deficient serum vitamin C levels. Taking these as reference values and assuming $80 \%$ power and $5 \%$ type 1 error with equal proportion, sample size was calculated to be 38 . Assuming $10 \%$ loss to follow-up, sample size was rounded off to 50 subjects.
The first 50 cases with PPROM between 28 and 36.6 weeks gestation, who fulfilled eligibility criteria, formed the study group. Control group was comprised of 50 patients with matching gestational age and body mass index, who were having uncomplicated pregnancies and intact membranes.

\section{Inclusion criteria}

Women, from 18 to 35 years of age, with singleton pregnancy between 28 and 36.6 weeks' gestational age, were included in the study.

\section{Exclusion criteria}

Women with anemia, diabetes mellitus, urinary tract infection, respiratory tract infection, vaginitis, polyhydramnios, history of smoking, and intake of micronutrients were excluded from the study.

Blood samples were collected initially from subjects in both groups, to establish the serum ascorbic acid and IL-6 levels, before the administration of any medication. Repeat samples for IL- 6 were taken after 48 hours of admission in the study group and at the onset of labor in the control group. Speculum examination was done in cases with PPROM to confirm the presence of amniotic fluid and inspect its color and odor. Fetal well-being was evaluated by ultrasound. After routine workup the study group subjects were managed as per hospital protocol.

\section{1) Serum IL-6}

Maternal $2 \mathrm{~mL}$ venous blood was drawn into sterile siliconecoated tubes, refrigerated at $5^{\circ} \mathrm{C}$, and centrifuged. Serum IL- 6 was measured in batches by a single investigator, using an enzyme-linked immune-sorbent assay kit (R\&D Systems, Minneapolis, MN, USA). This assay did not cross-react with other cytokines and had an inter- and intra-assay variation less than $5 \%$.

\section{2) Plasma ascorbic acid}

Maternal $2 \mathrm{~mL}$ venous blood samples were collected in heparinized vial to establish ascorbic acid baseline. Serum ascorbic acid levels were evaluated using spectrophotometry.

Placental membranes of both groups were sent after delivery for aerobic culture sensitivity testing. Samples were collected in a universal sterile container without any preservative or additive and sent immediately after collection. They were refrigerated at $4-8^{\circ} \mathrm{C}$, if there was a delay in sending 


\title{
Obstetrics \& Gynecology Science
}

\author{
Vol. 63 , No. 4, 2020
}

them to the microbiology laboratory. Samples were immediately processed in the microbiology laboratory. The placental membrane was well homogenized on a sterile Petri dish, using a bard-parker blade. The homogenized tissue was inoculated into 5\% sheep blood agar (McConkey agar plates and brain heart infusion [BHI] enrichment media). Gram stain was also done from the tissue. Inoculated plates were incubated for 48 hours at $37^{\circ} \mathrm{C}$ and inspected for zones of growth. If no growth was detected then subculture was prepared, using BHI media. The report was considered negative only if there is no growth after 72 hours. Any growth in any of the culture plates were further evaluated for identification and sensitivity testing. Manual identification was done by biochemical tests and supported in a few cases by Vitek 2.0.
Sensitivity testing of the isolates was done by disk diffusion test method following CLSI 2018.

Categorical variables are presented in number and percentage (\%) and continuous variables as mean \pm standard deviation and median. Comparison of quantitative variables was performed by unpaired $t$-test/Mann-Whitney test. Qualitative variables were compared using $\chi^{2}$ test /Fisher's exact test. $P$ value of $<0.05$ was considered statistically significant. The data was entered in MS Excel spreadsheet and analysis was done using SPSS version 25.0 (IBM Corp., Armonk, NY, USA). The accuracy of the multivariate predictors of gene mutations was measured using the area under the receiver operating characteristic (ROC) curve.

Table 1. Descriptive characteristics for preterm premature rupture of membranes (PPROM) and control groups

\begin{tabular}{|c|c|c|c|c|c|}
\hline Parameters & PPROM group & Control group & $P$-value & Adjusted OR (95\% Cl) & $P$-value \\
\hline Maternal age (yr) & $24.36 \pm 3.45$ & $24.08 \pm 3.33$ & $0.639^{a)}$ & - & - \\
\hline Gravidity (number) & $2.72 \pm 1.01$ & $2.24 \pm 1.04$ & $0.021^{a)}$ & Gravidity $\geq 3,1.79(0.73-4.37)$ & 0.190 \\
\hline Presence of preterm birth history & $18(36)$ & $5(10)$ & $0.002^{b)}$ & $4.36(1.39-13.71)$ & 0.010 \\
\hline Low socio-economic status & $34(68)$ & $17(34)$ & $0.003^{b)}$ & $3.56(1.48-8.59)$ & 0.050 \\
\hline Smoking & $3(6)$ & $2(4)$ & $0.646^{b)}$ & - & - \\
\hline Caesarean section & $14(28)$ & $10(20)$ & $0.349^{b)}$ & - & - \\
\hline Gestational age at birth (wk) & $34.00 \pm 1.54$ & $39.30 \pm 1.76$ & $0.000^{a)}$ & - & - \\
\hline Birth weight (kg) & $1.70 \pm 0.20$ & $3.10 \pm 0.41$ & $0.000^{\mathrm{a})}$ & - & - \\
\hline Neonatal morbidity and mortality & $37(74)$ & $12(24)$ & $0.000^{b)}$ & - & - \\
\hline
\end{tabular}

Data are shown as mean \pm standard deviation or number (\%).

$\mathrm{OR}$, odds ratio; $\mathrm{Cl}$, confidence interval.

a) Independent $t$-test; ${ }^{b)}$ Pearson $\chi^{2}$ test.

Table 2. Plasma ascorbic acid and interleukin (IL)-6 levels between preterm premature rupture of membranes (PPROM) group and control group

\begin{tabular}{|c|c|c|c|}
\hline Parameters & PPROM group $(n=50)$ & Control group $(n=50)$ & $P$-value \\
\hline Plasma ascorbic acid levels $(\mathrm{mg} / \mathrm{dL})$ at the time of enrolment & $0.60 \pm 0.35$ & $1.18 \pm 0.43$ & $0.000^{\mathrm{a})}$ \\
\hline Plasma IL-6 levels (pg/mL) at the time of enrolment & $18.18 \pm 5.94$ & $5.59 \pm 4.36$ & $0.000^{\mathrm{a})}$ \\
\hline Repeat plasma IL-6 levels (pg/mL) & $\begin{array}{c}34.32 \pm 7.31 \\
\text { (48 hr after admission) }\end{array}$ & $\begin{array}{l}\quad 7.36 \pm 3.67 \\
\text { (at the onset of labour) }\end{array}$ & - \\
\hline No. of patients with low plasma ascorbic acid ${ }^{\text {b) }}$ & $33(66)$ & $6(12)$ & $\left.0.000^{c}\right)$ \\
\hline No. of patients with high IL-6 ${ }^{b}$ & $39(78)$ & $3(6)$ & $0.000^{c}$ \\
\hline No. of placental membrane culture (+) & $37(74)$ & $3(0.06)$ & $0.000^{c}$ \\
\hline
\end{tabular}

Data are shown as mean \pm standard deviation or number (\%).

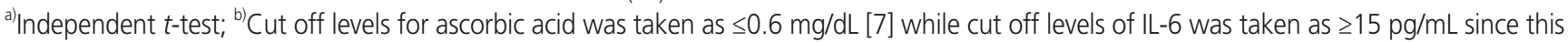
value predicted microbial invasion (confirmed on membrane culture sensitivity) of amniotic membranes in PPROM with high sensitivity and specificity; ${ }^{c}$ Pearson $\chi^{2}$ test. 


\section{Obstetrics \& Gynecology Science}

Sumedha Gupta, et al. Predictability of vit C and IL 6 levels for PPROM

\section{Results}

Descriptive characteristics of PPROM and control group are summarized in Table 1.

Univariate analysis showed patients, belonging to lower socioeconomic status and of higher gravida, were more prone to develop PPROM. Patients with a history of preterm birth were having five times higher risk of PPROM (odds ratio [OR], 5.06; 95\% confidence interval [CI], 1.70-15.05) in the present pregnancy. The mean value of plasma ascorbic acid was $0.60 \pm 0.35 \mathrm{mg} / \mathrm{dL}$ in PPROM group and $1.18 \pm 0.43 \mathrm{mg} / \mathrm{dL}$ in the control group at the time of enrollment at the same gestational age. Patients with low ascorbic acid levels were having 14 times higher risk of PPROM $(\mathrm{OR}, 14.23 ; 95 \% \mathrm{Cl}$, 5.06-40.04). Mean levels of plasma IL-6 at the time of enrollment in patients of PPROM group was higher $(18.18 \pm 5.94$ $\mathrm{pg} / \mathrm{mL})$ than control group $(5.59 \pm 4.36 \mathrm{pg} / \mathrm{mL})$. The difference was statistically significant $(P$-value $=0.000$ ) (Table 2 ). Its levels were directly proportional to the duration of leaking at admission; however, there was weak correlation ( $r^{2}$ value $\left.=0.16\right)$.

In our study, the cutoff level for ascorbic acid was $\leq 0.6 \mathrm{mg} /$ $\mathrm{dL}$ [7]; hence, predicting the chances of PPROM with sensitivity of $66 \%$ and specificity of $88 \%$ and ROC analysis showed an area under the curve (AUC) of $0.841(95 \% \mathrm{Cl}, 0.78-0.93)$. The cutoff level of IL-6 was $\geq 15 \mathrm{pg} / \mathrm{mL}$, and this value was found to predict microbial invasion (confirmed on membrane culture sensitivity tests) of amniotic membranes in PPROM, with a sensitivity of $85 \%$ and specificity of $86.67 \%$, and ROC analysis showed an AUC of $0.915(95 \% \mathrm{Cl}, 0.85-0.97)$ (Table 3 and Fig. 1).

After 48 hours of admission, repeat plasma IL-6 level rose

Table 3. The relationship between microbial invasion and interleukin (IL)-6 level

\begin{tabular}{|c|c|c|c|c|c|c|c|c|}
\hline $\begin{array}{l}\text { Laboratory } \\
\text { test }\end{array}$ & Microb & ion (+) & Microk & sion (-) & $\begin{array}{c}\text { Sensitivity } \\
(\%)\end{array}$ & $\begin{array}{c}\text { Specificity } \\
\text { (\%) }\end{array}$ & PPV (\%) & NPV (\%) \\
\hline$\overline{I L-6^{a)}}$ & $34^{b)}$ & 6) & $8^{\text {d) }}$ & $52^{\mathrm{e})}$ & 85 & 86.67 & 80.95 & 89.65 \\
\hline
\end{tabular}

n, number of subjects; PPV, positive predictive value; NPV, negative predictive value.

a) IL-6: No. of patients with high IL-6 level ( $\geq 15 \mathrm{pg} / \mathrm{mL})(\mathrm{n}=42) ;{ }^{\text {b) }}$ True positive; ${ }^{\text {c)}}$ False negative; ${ }^{\text {d) }}$ False positive; ${ }^{\text {e) }}$ True negative.

A

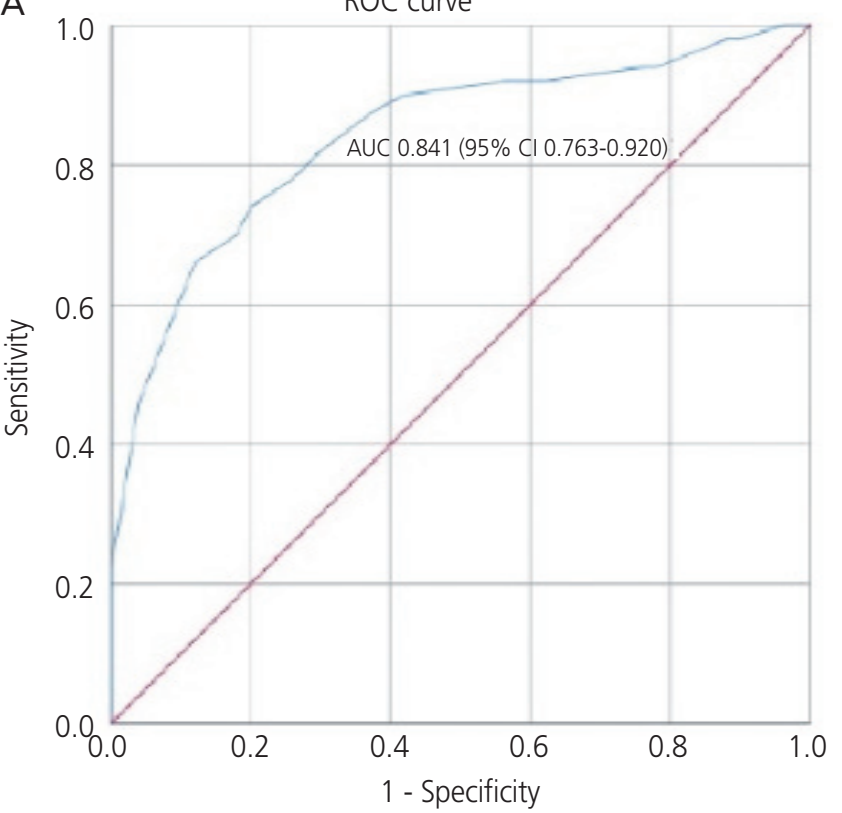

Diagonal segments are produced by ties.

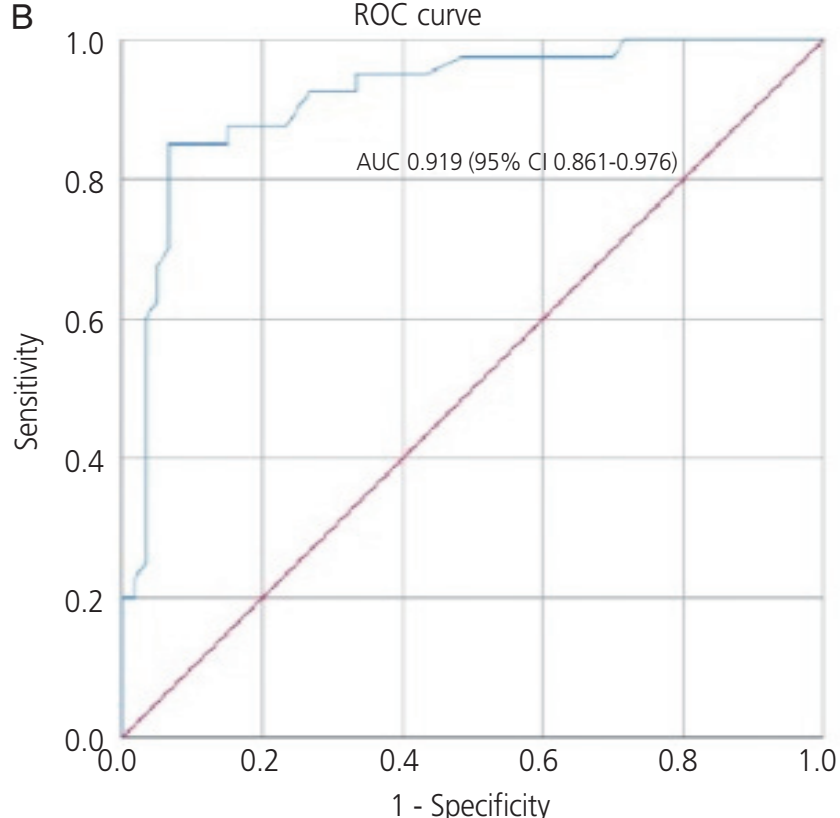

Diagonal segments are produced by ties.

Fig. 1. (A) Receiver operating characteristic (ROC) curve for serum vitamin $C$ as predictor of preterm premature rupture of membranes. (B) ROC curve for serum interleukin- 6 as predictor of microbial invasion of placental membranes. AUC, area under the curve; $\mathrm{Cl}$, confidence interval. 


\section{Obstetrics \& Gynecology Science}

Vol. 63, No. 4, 2020

from $18.18 \pm 5.94$ to $34.32 \pm 7.31 \mathrm{pg} / \mathrm{mL}$, the difference being statistically significant $(P<0.000)$. However, the mean plasma level of IL-6 in controls rose from $5.59 \pm 4.36$ at admission to $7.36 \pm 3.67$ at the onset of labor. Membrane culture tested positive, suggesting microbial invasion in a total of 40 cases, of which 37 cases presented PPROM. Only 3 cases without PPROM had evidence of microbial invasion.

There was no difference in the mode of delivery in both the groups but statistically significant difference in birthweight of newborns existed. Neonatal morbidity developed in higher proportion in the PPROM group. Septicemia and conjunctivitis developed in $28 \%$ and $6 \%$ of babies in the PPROM group, while conjunctivitis developed in $4 \%$ in the control group. Respiratory distress syndrome was present in $24 \%$ of patients in the PPROM group, while it was present in only $10 \%$ of patients in the control group. Jaundice contributed equally $(10 \%)$ to fetal morbidity in both groups. This proportional difference in the neonatal morbidity showed high statistical significance $(P$-value $=0.001)$. Neonatal mortality was present in $6 \%$ of the patients from the PPROM group while none in the control group. Among women with low plasma vitamin C levels, $90.9 \%$ of neonates had one or other morbidity.

\section{Discussion}

Primary outcome (Table 2) revealed mean plasma level of ascorbic acid was significantly low while IL-6 level was high at the time of enrollment in the PPROM group. There was significant difference in the number of women with low serum ascorbic acid and high IL- 6 level at the time of enrollment among PPROM and control group. IL- 6 levels rose significantly high after 48 hours of admission among cases while the rise was moderately high among controls, comparing values during enrollment and at onset of labor. Secondary analysis revealed there was a significant difference between PROM group subjects and controls in terms of socioeconomic status, gravidity, history of preterm birth, birthweight, and perinatal morbidity (Table 1). However, multi-logistic analysis (Table 4) revealed that women having a history of preterm delivery and those with high serum IL-6 level at the time of enrollment were only at a significant high-risk of PPROM.

Vitamin $C$ is a micronutrient that directly stimulates collagen synthesis by activating multiple genes. It is considered that it maintains the equilibrium between synthesis, stability, and degradation of collagen, which in turn may reduce the incidence of PPROM [8]. It also acts as an antioxidant and this role of vitamin $C$ is most evident during periods of elevated oxidative stress. Phagocytes are activated because of many infections and they release ROS, which play a role in elimination of virus and bacteria [9]. These ROS are, however, not without side effects and may be harmful to the host cells, thus, playing a probable role in the pathogenesis of microbial invasion [10]. Ascorbic acid is assumed to play a role in protecting host cells against the detrimental effects of ROS, released by phagocytes, and hence, its levels are decreased during infections [11]. Therefore, ascorbic acid levels may be decreased in most infections, occurring after PPROM, thus acting as a cause as well as effect. This was evident in our study too. Ascorbic acid levels were significantly lower in the PPROM group, which might have triggered the occurrence of PPROM. Otherwise primary microbial invasion of placental membranes, as evident from the high IL- 6 levels at the time of enrollment, was the cause for the decreasing level of ascorbic acid in PPROM group. Hence, IL-6 and ascorbic acid both can be utilized as marker for PPROM and

Table 4. Significant predictive factors for preterm premature rupture of membranes based on logistic regression test results

\begin{tabular}{llc}
\hline Factors & P-value & Adjusted OR (95\% Cl) \\
\hline Low socioeconomic status & 0.87 & $1.12(0.25-4.84)$ \\
Presence of history of preterm birth & $0.01^{\text {a) }}$ & $7.93(1.51-41.72)$ \\
Plasma ascorbic acid at the time of enrolment & 0.53 & $2.13(0.19-23.48)$ \\
Plasma IL-6 at the time of enrolment & $0.00^{\text {a) }}$ & $1.41(1.11-1.80)$ \\
No. of patients with low serum ascorbic acid at the time of enrolment & 0.50 & $0.38(0.03-6.68)$ \\
No. of patients with high IL-6 at the time of enrolment & 0.59 & $2.45(0.07-50.63)$ \\
\hline
\end{tabular}

$\mathrm{IL}$, interleukin; $\mathrm{OR}$, odds ratio; $\mathrm{Cl}$, confidence interval.

a) Significant $P$-value. 


\section{Obstetrics \& Gynecology Science}

Sumedha Gupta, et al. Predictability of vit C and IL 6 levels for PPROM

microbial invasion. Siega-Riz et al. [12] found a relationship between low vitamin $C$ intake and an increased risk of PPROM (adjusted OR, 1.03; 95\% Cl, 0.90-1.17). Sharma and Mehta [3] observed low vitamin C concentration in patients with PPROM and an inverse relationship between the duration of the rupture of membranes and vitamin $C$ levels with a significant increase in maternal morbidity, neonatal morbidity, and mortality. This inverse relationship evidenced in study by Sharma and Mehta [3] reinforces our findings though they did not measure any inflammatory marker in serum. However, the results of studies, defining the therapeutic role of the micronutrient are not encouraging. Steyn et al. [13] observed no significant difference in the occurrence of preterm labor with ascorbic acid supplementation $(250 \mathrm{mg} /$ day) and concluded that the therapeutic effects of vitamin C may be evident only in patients with deficiency of the micronutrient. Spinnato et al. [14] observed that high-dose vitamin C (1,000 mg/day) caused an increase in the incidence of PROM in a randomized double-blind placebo-control trial and warned against the empirical use of the micronutrient. Mercer et al. [15] observed that at high doses, vitamin C may cause collagen degradation and apoptosis, thus questioning the beneficial role of vitamin $C$ in the prevention of PPROM. They concluded that vitamin $C$ has no role in the remodeling process of normal fetal membrane, which may lead to weakening and rupture at term. However, a randomized doubleblind placebo-controlled trial proved the effectiveness of vitamin C at a dose of $100 \mathrm{mg} /$ day in preventing PPROM [16]. Hence, the therapeutic role of the micronutrient needs to be elucidated in deficient and sufficient subjects to evaluate bona fide effects. The dose has to be optimized after adjusting to plasma levels and duration of gestation, so that the inadvertent harmful effects, if any, are avoided.

Our body cannot synthesize vitamin C and diet is the main source of this micronutrient [13]. Inadequate intake is hence the possible cause for the prevalence of deficiency of vita$\min C$ found in our study population. An imprudent attitude and casual approach in terms of clinical visits, drug intake, physical labor and diet among Indian women towards higher order pregnancies, could be a result from the limitations of social framework, prevalent in the country. This may also explain the observation made by us and in other studies $[17,18]$ of higher association of PPROM with low socioeconomic status. A history of prior preterm delivery or PPROM was seen in a greater proportion in the study group, which is consistent with results of other studies $[19,20]$. Negara et al. [21] observed that there was stronger p53 expression and stronger apoptotic index in the PROM group than in the PROM-free group. This genetic link may explain the occurrence of repeated events of rupture of membranes in a woman. Our study found that there was no difference in the mode of delivery in the study and the control group, like Sharma and Mehta [3] did, while other studies [22,23] reported higher rate of caesarean deliveries among women with PPROM. Poor perinatal outcomes were observed in our study group, consistent with results of another study [24].

In conclusion our study demonstrates that low serum vitamin C levels alone can poorly predict the occurrence of PPROM. However, when this is complemented with elevated IL-6 level after 28 weeks, susceptibility to PPROM increases, given the subject has had prior history of such complication.

Measuring serum levels of IL-6 and vitamin C will assist in counselling and prognosticating patients with anticipated rupture of membranes. Optimizing the serum levels of ascorbic acid before conception and during pregnancy, particularly in those with micronutrient deficiency or a history of PPROM, can be a rewarding therapeutic intervention for the prevention of this obstetric complication.

\section{Acknowledgements}

We thank Dr. Anita Rani, Professor, Department of Biochemistry and Dr. Annapurna Parida, Senior Resident, Department of Microbiology, VMMC and Safdarjung Hospital, for helping us conduct this study.

\section{Conflict of interest}

No potential conflict of interest relevant to this article was reported.

\section{Ethical approval}

This study was approved by the Institute Ethics Committee (S.No.IECNMMC/SJH/Thesis/October-2015). All tests were done free of cost in our hospital and were provided by the Government of India. 


\section{Obstetrics \& Gynecology Science}

Vol. 63, No. 4, 2020

\section{Patient consent}

Informed written consent was taken from all subjects before enrolling them in the study.

\section{References}

1. Hartling L, Chari R, Friesen C, Vandermeer B, LacazeMasmonteil T. A systematic review of intentional delivery in women with preterm prelabor rupture of membranes. J Matern Fetal Neonatal Med 2006;19:177-87.

2. ACOG Committee on Practice Bulletins-Obstetrics. ACOG practice bulletin No. 80: premature rupture of membranes. Clinical management guidelines for obstetrician-gynecologists. Obstet Gynecol 2007;109:100719.

3. Sharma R, Mehta S. Ascorbic acid concentration and preterm premature rupture of membranes. J Obstet Gynaecol India 2014;64:417-20.

4. Qiu X, Zhang L, Tong Y, Qu Y, Wang H, Mu D. Interleukin-6 for early diagnosis of neonatal sepsis with premature rupture of the membranes: a meta-analysis. Medicine (Baltimore) 2018;97:e13146.

5. Gulati S, Bhatnagar S, Raghunandan C, Bhattacharjee J. Interleukin-6 as a predictor of subclinical chorioamnionitis in preterm premature rupture of membranes. Am J Reprod Immunol 2012;67:235-40.

6. Osaikhuwuomwan JA, Okpere EE, Okonkwo CA, Ande $A B$, Idogun ES. Plasma vitamin $C$ levels and risk of preterm prelabour rupture of membranes. Arch Gynecol Obstet 2011;284:593-7.

7. Mayo Medical Laboratories. Ascorbic acid (vitamin C), plasma [Internet]. Rochester (MN): Mayo Medical Laboratories; c2020 [cited 2020 Jun 4]. Available from: https://www.mayomedicallaboratories.com/test-catalog/ Overview/42362.

8. Osaikhuwuomwan JA. Preterm rupture of membranes: the vitamin $\mathrm{C}$ factor. Benign J Postgrad Med 2010;12:60-8.

9. Harpsøe MC, Nielsen NM, Friis-Møller N, Andersson M, Wohlfahrt J, Linneberg $A$, et al. Body mass index and risk of infections among women in the Danish national birth cohort. Am J Epidemiol 2016;183:1008-17.

10. Akaike T. Role of free radicals in viral pathogenesis and mutation. Rev Med Virol 2001;11:87-101.

11. Hemilä H. Vitamin C and infections. Nutrients 2017:9:339.

12. Siega-Riz AM, Promislow JH, Savitz DA, Thorp JM Jr, McDonald T. Vitamin C intake and the risk of preterm delivery. Am J Obstet Gynecol 2003;189:519-25.

13. Steyn PS, Odendaal HJ, Schoeman J, Stander C, Fanie N, Grové D. A randomised, double-blind placebo-controlled trial of ascorbic acid supplementation for the prevention of preterm labour. J Obstet Gynaecol 2003;23:150-5.

14. Spinnato JA 2nd, Freire S, Pinto e Silva JL, Rudge MVC, Martins-Costa S, Koch MA, et al. Antioxidant supplementation and premature rupture of the membranes: a planned secondary analysis. Am J Obstet Gynecol 2008;199:433.e1-433.e8.

15. Mercer BM, Abdelrahim A, Moore RM, Novak J, Kumar D, Mansour JM, et al. The impact of vitamin C supplementation in pregnancy and in vitro upon fetal membrane strength and remodeling. Reprod Sci 2010;17:685-95.

16. Casanueva E, Ripoll C, Tolentino M, Morales RM, Pfeffer F, Vilchis $P$, et al. Vitamin $C$ supplementation to prevent premature rupture of the chorioamniotic membranes: a randomized trial. Am J Clin Nutr 2005;81:859-63.

17. Ferguson SE, Smith GN, Salenieks ME, Windrim R, Walker MC. Preterm premature rupture of membranes. Nutritional and socioeconomic factors. Obstet Gynecol 2002;100:1250-6.

18. Hackenhaar AA, Albernaz EP, da Fonseca TMV. Preterm premature rupture of the fetal membranes: association with sociodemographic factors and maternal genitourinary infections. J Pediatr (Rio J) 2014;90:197-202.

19. Shen TT, DeFranco EA, Stamilio DM, Chang JJ, Muglia LJ. A population-based study of race-specific risk for preterm premature rupture of membranes. Am J Obstet Gynecol 2008;199:373.e1-373.e7.

20. Mercer BM, Goldenberg RL, Meis PJ, Moawad AH, Shellhaas C, Das A, et al. The preterm prediction study: prediction of preterm premature rupture of membranes through clinical findings and ancillary testing. The National Institute of Child Health and Human Development Maternal-Fetal Medicine Units Network. Am J Obstet Gynecol 2000;183:738-45.

21. Negara KS, Prajawati NL, Surya GP, Suhendro S, Arijana K, Tunas K. Protein 53 (P53) expressions and apoptotic 


\section{Obstetrics \& Gynecology Science}

Sumedha Gupta, et al. Predictability of vit C and IL 6 levels for PPROM

index of amniotic membrane cells in the premature rupture of membranes. Open Access Maced J Med Sci 2018;6:1986-92.

22. Tavassoli F, Ghasemi M, Mohamadzade A, Sharifian J. Survey of pregnancy outcome in preterm premature rupture of membranes with amniotic fluid index $<5$ and 25. Oman Med J 2010;25:118-23.

23. Kayiga $H$, Lester F, Amuge PM, Byamugisha J, Autry AM.
Impact of mode of delivery on pregnancy outcomes in women with premature rupture of membranes after 28 weeks of gestation in a low-resource setting: a prospective cohort study. PLoS One 2018;13:e0190388.

24. Elimian A, Verma U, Beneck D, Cipriano R, Visintainer $P$, Tejani N. Histologic chorioamnionitis, antenatal steroids, and perinatal outcomes. Obstet Gynecol 2000;96:333-6. 